\title{
Comparison of the Attractive Forces of Dental Magnetic Attachments Fabricated by Cast- and Direct-Bonding Techniques
}

\author{
Fujio Tsuchida, DMD, PhD, Yuko Suminaga, DMD, Norio Takishin, DDS, PhD, \\ Toshio Hosoi, DDS, PhD, and Akira Ohshima, DMD, PhD \\ Department of Removable Prosthodontics, Tsurumi University School of Dental Medicine, Yokohama, Japan
}

\section{Clinical significance}

A magnetic attachment can serve as an extremely effective tool for the design of prosthesis. It is highly important to identify the alterations in the attractive force of magnetic attachments that would be affected by the fabrication methods.

\begin{abstract}
Purpose: This study evaluated the effect of the method of keeper fabrication on the attractive force of the magnetic attachment.

Methods: The attractive forces of the keepers of a $\mathrm{Hy}^{-}$ per Slim 3513 magnetic attachment system (Neomax Material Co. Ltd., Osaka, Japan) and Physio magnetic attachment system 35 (Nissin Co. Ltd., Kyoto, Japan) were measured. Specimens were prepared by the castbonding $(n=5)$ and direct-bonding $(n=5)$ techniques. The attractive force was measured using a digital force gauge (FGC-1, Nidec-Shimpo Corporation, Kyoto, Japan). The attractive force was statistically analyzed by the $t$-test at a significance level of $\alpha=0.05$.

Results: The direct-bonding technique yielded a keeper with significantly greater attractive force than that of the keeper obtained by the cast-bonding technique $(P<0.05)$.

Conclusion: Measurement of the attractive force of magnetic attachments fabricated by two different techniques revealed that the magnetic attachment fabricated by direct bonding possessed a significantly greater attractive force than that fabricated by cast bonding. Our results and those of previous studies suggest that the flatness and smoothness of the keeper surface may influence the attractive force of the magnetic attachment.
\end{abstract}

Key words: dental magnetic attachment, keeper, direct-bonding technique, cast-bonding technique, attractive force

\section{Corresponding to: Dr Fujio Tsuchida}

Department of Removable Prosthodontics, Tsurumi

University School of Dental Medicine

2-1-3 Tsurumi, Tsurumi-ku, Yokohama 230-8501, Japan

Tel: +81-45-581-1001, Fax: +81-45-573-9599

E-mail: tsuchida-f@tsurumi-u.ac.jp

\section{Introduction}

Generally, the greatest attractive force of dental magnetic attachments is influenced by the flatness of the keeper surface. ${ }^{1}$ There are two techniques to fabricate a coping, including the keepers, on the root of a tooth in order to secure the dental magnetic attachments. One is the conventional and widely used cast-bonding technique. $^{2}$ The other is the recently developed direct-bonding technique in which the keepers are cemented to the coping with resin cement. While the keeper surface might be altered due to the heating and polishing during cast bonding, it would not be affected during direct bonding. ${ }^{3}$

The attractive force of the fabricated magnetic attachment using direct bonding may closely replicate the force of the original attachment. The purpose of this study was to compare the attractive force of the keepers fabricated by the castand direct-bonding techniques.

\section{Materials and methods}

The copings for cast bonding were fabricated from the keepers of the Hyper Slim $3513 \mathrm{mag}^{-}$ netic attachment system $(0.8 \mathrm{~mm}$ thick, $3.5 \mathrm{~mm}$ in diameter; Neomax Co. Ltd., Osaka, Japan) (Fig. 1). Those for direct bonding were fabricated from the keepers of the Physio magnetic attachment system $(0.8 \mathrm{~mm}$ thick, $3.5 \mathrm{~mm}$ in diameter; Nissin Co. Ltd., Kyoto, Japan). For the directbonding technique, an acrylic pattern was used to fabricate the keeper housing. The keeper housing was manufactured such that it was $0.1 \mathrm{~mm}$ larger than the keeper (Fig. 2). In direct bonding, a keeper that was not cemented to the coping (Physio magnetic attachment system) was used as a control. 


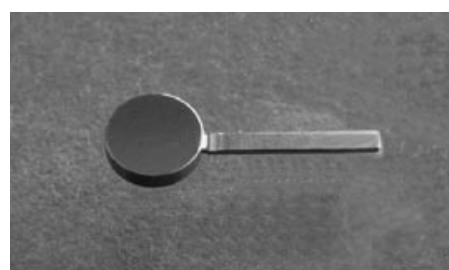

Fig. 1 The keeper of the Hyper Slim 3513 magnetic attachment system used for cast-bonding. (0.8 $\mathrm{mm}$ thick, $3.5 \mathrm{~mm}$ in diameter)

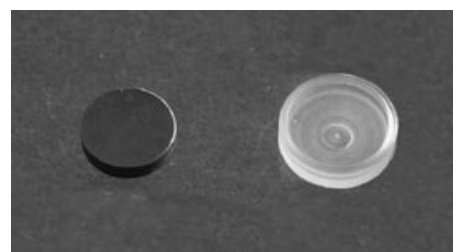

Fig. 2 The keeper of the Physio magnetic attachment system used for direct-bonding $(0.8 \mathrm{~mm}$ thick, $3.5 \mathrm{~mm}$ in diameter) and the acrylic pattern used to fabricate the keeper housing.

\section{Preparation of abutment tooth models}

To fabricate the coping along with the keeper, five abutment tooth models of the mandibular right canine (original root form, A50AN-438, Nissin) were used in both the techniques. The tooth models were mounted on an acrylic plinth $(15 \times 15$ $\times 4 \mathrm{~mm}$ ), and the dowel length was $5 \mathrm{~mm}$ (Fig. 3).

\section{Cast-bonding technique}

Wax patterns (Inlay wax medium, GC, Tokyo, Japan) of the coping along with the keeper were prepared according to conventional laboratory procedures. The casting ring $\left(\phi 34-\mathrm{R} 1, \mathrm{KDF}, \mathrm{To}^{-}\right.$ kyo, Japan) was lined with a ring liner (New Casting Liner No.1, GC, Tokyo, Japan). The wax pattern was invested in the center of the ring and cast with $\mathrm{Au}-\mathrm{Ag}-\mathrm{Pd}$ alloy (Castwell $\mathrm{MC}$, GC, Tokyo, Japan) in a vacuum casting machine (CASCOM, KDF, Tokyo, Japan) that was maintained at $700^{\circ} \mathrm{C}$ for 30 minutes to allow burnout. After all the specimens were bench-cooled to room temperature, they were removed from the casting ring. The keeper surface was polished with \#4000 ultra-precision finishing film (Sumitomo 3M Ltd., Tokyo, Japan) that was glued to a glass slab. Films of varying particle sizes were used to polish the keeper surface. Consequently, the \#4000 ultra-precision finishing film imparted a mirror-like finish to the keeper surface. As far as possible, the keeper surface was maintained parallel to the glass slab, and it was polished by

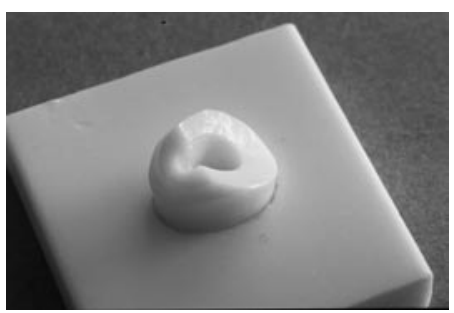

Fig. 3 An original abutment tooth model of the mandibular right canine attached to a plinth prepared from acrylic resin.

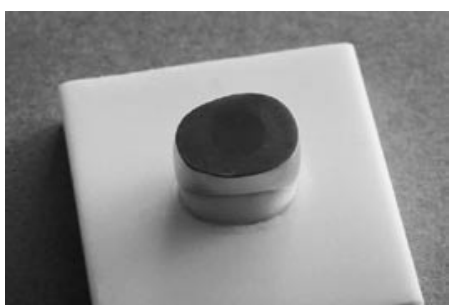

Fig. 4 The coping prepared by the cast-bonding technique; it was polished with \#4000 ultra-precision finishing film.

sliding it on the glass slab. As a standard, the keeper surface required polishing till a mirrorlike finish was achieved (Fig. 4).

\section{Direct-bonding technique}

For the direct bonding technique, the coping was waxed-up by using the housing pattern and cast under the same conditions as those used for the cast-bonding technique. The housing pattern was set using a dental surveyor. After the inside surface of the housing was abraded with airborne particles, i.e., $50-\mu \mathrm{m}$ alumina oxide particles (air pressure, $2 \sim 3 \mathrm{kgf} / \mathrm{cm}^{2}$ ), the top of the housing edge was polished in the same manner as that used in the cast-bonding technique. A metal conditioner (Alloy Primer, Kuraray Medical Inc., Tokyo, Japan) was primed on the bonding surface, and the keeper was then cemented on the coping housing by using resin cement (Panavia Fluoro cement, Kuraray Medical Inc., Tokyo, Japan), according to the procedure reported by Hirano et $\mathrm{al}^{4}$ (Fig. 5).

\section{Measurement of the attractive force}

The attractive forces of the magnetic attachments fabricated by cast and direct bonding were measured with a digital force gauge (FGC-1; Nidec-Shimpo Corporation, Kyoto, Japan) (Fig. 6). The coping with the keeper could be set up using this equipment, allowing measurement of the attractive force produced by the magnetic attach- 


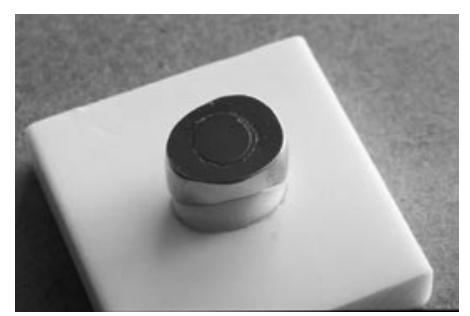

Fig. 5 The coping prepared by the direct-bonding technique; the keeper was cemented in the housing by using Panavia Fluoro cement.

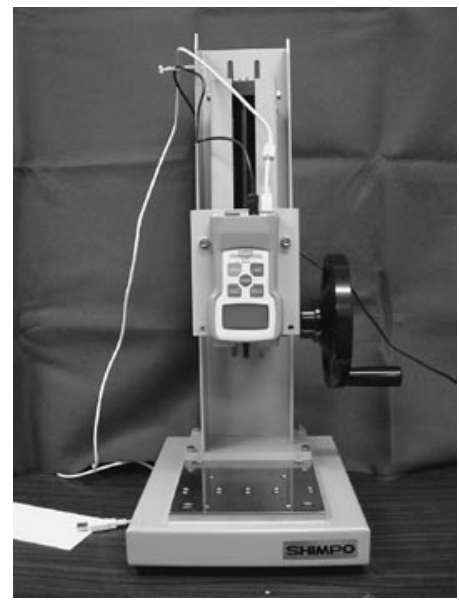

Fig. 6 The digital force gauge used to measure the attractive force (FGC-1, Nidec-Shimpo).

ments. The attractive force between the original magnetic assembly and the original keeper was measured and used as the control. The attractive forces of the magnetic attachments fabricated by the cast and direct-bonding techniques were statistically analyzed using the $t$-test (SPSS ver. 12 , SPSS Japan Inc., Tokyo, Japan) at a significance level of $\alpha=0.05$.

\section{Results}

The attractive forces of the magnetic attachments fabricated by the cast-bonding and directbonding techniques are shown in Figure 7; the mean attractive forces produced by them were $4.81 \pm 0.052 \mathrm{~N}$ and $4.98 \pm 0.030 \mathrm{~N}$, respectively. On the other hand, the mean attractive force of the control was $5.16 \pm 0.0070 \mathrm{~N}$. Thus, the mean attractive forces of both the fabricated magnetic attachments were significantly lower than that of the control $(P<0.05)$.

However, the mean attractive force of the magnetic attachment fabricated by direct bonding

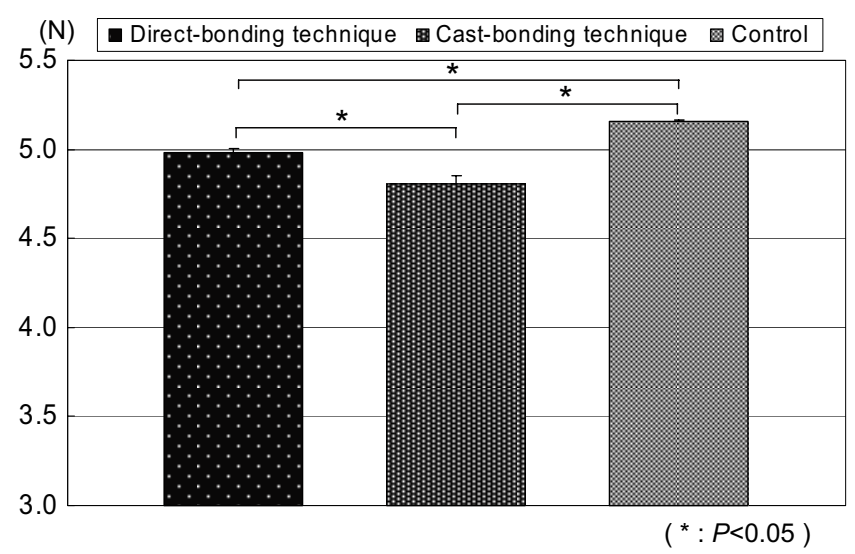

Fig. 7 Comparison of the attractive forces of keepers fabricated using the cast-bonding and direct-bonding techniques. ( $t$-test, $\left.{ }^{*} P<0.05\right)$

was significantly greater than that of the attachment fabricated by cast bonding $(P<0.05)$.

\section{Discussion}

Factors that affect the attractive force of magnetic attachments include heating of the keeper during cast bonding, the planar accuracy of the keeper surface, the presence of a gap in the magnetic assembly, and the type of investment material used. To minimize the influence of these factors, the direct-bonding technique was developed. ${ }^{4}$ The direct-bonding technique provides an excellent planar accuracy of the coping and keeper. This planar relation of the polished keeper surface greatly influences the attractive force. A previous study reported the results obtained during analysis on the change in the 3 -dimensional shape of keepers that were fabricated by the cast- and direct-bonding techniques. ${ }^{1}$ The keepers fabricated by direct bonding were significantly flatter than those fabricated by cast bonding; the surfaces of the latter had a central bulge. In this study, the attractive force of the keeper fabricated by direct bonding was significantly greater than that of the keeper fabricated by cast bonding. The convex center of the keeper may not be the only reason for the lower attractive force. Any gaps between the magnet and the keeper would also noticeably decrease the attractive force.$^{5-7}$ In addition, the planar accuracy of the keeper's absorption side can also decrease the attractive force. The greatest attractive force is obtained when the magnetic assembly and the keeper adhere completely, i.e., without any gaps. The keepers fabricated by 
direct bonding were nearly flat because this technique does not involve casting procedures. The direct-bonding technique does not comprise casting shrinkage because the keeper is cemented in the coping housing using resin cement. The castbonding technique includes casting with keepers that are convex as a result of casting shrinkage. The direct-bonding technique resulted in a lower attractive force than that obtained for the control. However, a value similar to the original attractive force of the magnetic attachment was obtained because the flat surface of the keeper was maintained. In contrast, direct bonding does not accommodate an attachment of the same size as that in cast bonding because it requires keeper housing. The results of this study suggest that it is necessary to select the appropriate method to fabricate keeper coping, depending on the clinical situation.

\section{Conclusion}

Measurement of the attractive forces of magnetic attachments fabricated by the direct- and castbonding techniques revealed that direct bonding yielded an attachment with a significantly greater attractive force $(P<0.05)$.

Within the limitations of the experimental study, the results suggested that keepers fab- ricated by the direct-bonding method possess significantly larger attractive forces than those fabricated by casting.

\section{References}

1. Suminaga Y, Tsuchida F, Takishin N et al. Surface analysis of keepers on dental magnetic attachments: Comparison of cast-bonding technique and direct-bonding technique. Prosthodont Res Pract 3: 62-68, 2004.

2. Tanaka Y. Dental magnetic attachment, 122-170, Tokyo:Ishiyaku, 1992. (in Japanese)

3. Honda Y. Clinics of magnetic attachment, Change from casting to DB method. J Jpn Prosthodont Soc 47 109: 40, 2003.

4. Hirano T, Sugiyama K, Mizuno Y et al. The laboratory procedure of magnetic attachment by direct bonding method. J Jpn Magn Dent 12: 40-45, 2003. (in Japanese)

5. Mizutani H, Kadoyama N, Ai M et al. Influences of dental magnetic keeper area and the setting location on attractive force. J Jpn Magn Dent 1:61-70, 1992. (in Japanese)

6. Akaltan F, Can G. Retentive characteristics of different dental magnetic systems. J Prosthet Dent 74: 422-427, 1995.

7. Tegawa Y, Kinouchi Y. Influences on attractive force in different clinical uses of a cup yoke magnetic attachment. J Jpn Magn Dent 5: 31-38, 1996. (in Japanese) 